\title{
Propuesta para formular una estrategia de contingencia en Ortopedia y Traumatología frente a la pandemia de COVID-19
}

\author{
Guillermo A. Ricciardi, Martín Pérez, Santiago Scalambro, Gustavo Ricciardi, Gabriel Carrioli, \\ Fernando Locaso, Daniel Ricciardi \\ Servicio de Ortopedia y Traumatología, Hospital General de Agudos “Dr. Teodoro Álvarez”, Ciudad Autónoma de Buenos Aires, \\ Argentina
}

\section{RESUMEN}

Objetivo: Describir el proceso para la formulación e implementación de una estrategia de contingencia de un servicio público de Ortopedia y Traumatología frente a la pandemia de COVID-19. Materiales y Métodos: Estudio descriptivo del proceso para la formulación e implementación de una estrategia de contingencia de la pandemia de COVID-19 en un Servicio de Ortopedia y Traumatología del sistema público de salud de la Ciudad Autónoma de Buenos Aires, entre el 12 de marzo y el 20 de abril de 2020 , período en el cual fue declarada la emergencia sanitaria y el alerta amarilla en nuestro medio frente a la posibilidad inminente de un potencial aumento exponencial de casos de COVID-19. A cargo de un Comité de Pandemia de Ortopedia y Traumatología, el proceso fue dividido en tres instancias: planificación, implementación y control. Resultados: Basados en la normativa nacional, 17 artículos internacionales, 13 guías de sociedades científicas y 17 artículos de recomendaciones de la Organización Mundial de la Salud y los Centros para el Control y la Prevención de Enfermedades de los Estados Unidos. Definimos 7 áreas de trabajo: 1) Sala de internación, 2) Quirófano, 3) Consultorios externos, 4) Servicio de Urgencias, 5) Insumos, 6) Cuidado del personal de salud, 7) Docencia e investigación. Conclusión: Nuestro trabajo aporta una propuesta para el proceso de desarrollo de una estrategia de contingencia frente a la pandemia de COVID-19. Organizarse, capacitarse y protegerse como equipo son sus pilares fundamentales.

Palabras clave: Pandemia; COVID-19; estrategia de contingencia; Ortopedia y Traumatología.

Nivel de Evidencia: IV

Contingency plan development proposal for a Department of Orthopedics and Traumatology in the setting of the COVID-19 pandemic

\begin{abstract}
Objective: To describe the writing and implementing process of a contingency plan for a public healthcare Department of Orthopedics and Traumatology in the setting of the COVID-19 pandemic. Materials and Methods: Descriptive study of the writing and implementing process of a COVID-19 contingency plan at a Department of Orthopedics and Traumatology of the Buenos Aires City Public Healthcare System, during the period between March 12 and April 20, 2020, when the public health emergency and yellow alert was declared in our environment amid the imminent possibility of a potentially exponential increase of COVID-19 cases. Under the Pandemic Committee of Orthopedics and Traumatology supervision, the process was divided into 3 instances: planning, implementation, and control. Results: Data source: federal regulations, 17 international articles, 13 scientific association guidelines, and 17 articles of recommendations from the World Health Organization (WHO) and the United States Center for Disease Control and Prevention (CDC). Seven sectors were defined: 1) Hospitalization room; 2) Operating room; 3) Outpatient clinic; 4) Emergency Department; 5) Supplies; 6) Healthcare personnel control; 7) Education and research. Conclusion: We provide a writing and implementing process for the development of a contingency plan in the setting of the COVID-19 pandemic. Organizing, training and protecting yourselves as a team constitutes the plan fundamental pillars.
\end{abstract}

Key words: Pandemic; COVID-19; contingency plan; Orthopedics; Traumatology.

Level of Evidence: IV

Recibido el 23-4-2020. Aceptado luego de la evaluación el 6-5-2020 • Dr. GUILLERMO A. RICCIARDI • guillermoricciardi@gmail.com

https://orcid.org/0000-0002-6959-9301

Cómo citar este artículo: Ricciardi GA, Pérez M, Scalambro S, Ricciardi G, Carrioli G, Locaso F, Ricciardi D. Propuesta para formular una estrategia de contingencia en Ortopedia y Traumatología frente a la pandemia de COVID-19. Rev Asoc Argent Ortop Traumatol 2020;85(3):283-294. https://doi.org/10.15417/issn. 1852-7434.2020.85.3.1106 


\section{INTRODUCCIÓN}

El 11 de marzo de 2020, el director general de la Organización Mundial de la Salud declaró el estado de pandemia por COVID-19, enfermedad producida por un nuevo coronavirus surgido en la ciudad de Wuhan (Provincia de Hubei, China) ${ }^{1-3}$ El 5 de marzo de 2020 se registró el primer caso confirmado de COVID-19 en la Argentina y, una semana después, se declaró la emergencia sanitaria en nuestro país. ${ }^{4-6}$ Frente al alerta amarilla instaurada en los hospitales de la Ciudad Autónoma de Buenos Aires (CABA), los diferentes actores del sistema de salud nos vimos obligados a una reestructuración sanitaria en el marco del Plan Operativo de Preparación y Respuesta al COVID-19 del Ministerio de Salud de la Nación. ${ }^{7}$ Recibimos directivas y protocolos de atención del Ministerio de Salud de la CABA que reglamentan la contención y el abordaje de pacientes con sospecha y confirmación de COVID-19. ${ }^{8-12}$ Sin embargo, estas medidas resultaron insuficientes para definir los lineamientos del espectro de actividades que desarrolla el Servicio de Ortopedia y Traumatología.

Por tal motivo, los médicos especialistas en Ortopedia y Traumatología nos enfrentamos con un desafío sin precedentes en nuestro medio: establecer una estrategia de contingencia en la pandemia. Afortunadamente, contamos con la experiencia de países de Asia, Europa y América del Norte. Publicaciones y guías de sociedades científicas coinciden en sostener la capacidad quirúrgica frente a urgencias traumatológicas y ortopédicas como prioridad. Es también relevante contemplar las acciones en todos los escenarios de nuestra especialidad (sala de internación, consultorios externos, quirófano y guardia), promover la capacitación y el entrenamiento en el uso de elementos de protección personal y en funciones no traumatológicas que eventualmente nos puedan ser requeridas. ${ }^{13-23}$

Pero ¿cómo accedemos a esa estrategia frente al dinamismo de la pandemia, el estrés, la incertidumbre que produce y la inexperiencia que tiñe a todos los profesionales del Servicio sin distinción de jerarquías?

Los autores reunimos, en este trabajo, información sobre el proceso para formular e implementar una estrategia de contingencia en un Servicio de Ortopedia y Traumatología de un hospital del sistema público de salud.

\section{OBJETIVO}

Describir el proceso para la formulación e implementación de una estrategia de contingencia de un servicio público de Ortopedia y Traumatología frente a la pandemia de COVID-19.

\section{MATERIALES Y MÉTODOS}

Realizamos un estudio descriptivo del proceso para la formulación e implementación de una estrategia de contingencia de la pandemia de COVID-19 en un Servicio de Ortopedia y Traumatología del sistema público de salud de la CABA, entre el 12 de marzo y el 20 de abril de 2020, período en el cual fue declarada la emergencia sanitaria y el alerta amarilla en nuestro medio, frente a la posibilidad inminente de un potencial aumento exponencial de casos de COVID-19.

\section{Análisis externo: etapa de la pandemia}

$\mathrm{Al}$ comienzo de nuestro trabajo, los casos diagnosticados en nuestro país eran 31 ( 1 fallecido) y estaba descrita la transmisión local a contactos estrechos ${ }^{4-6} \mathrm{El} 20$ de marzo de 2020, el gobierno declaró el aislamiento social, preventivo y obligatorio. ${ }^{24}$ Los autores consideramos clave en el desarrollo de la estrategia estadificar la pandemia para guiar la toma de decisiones y los tiempos para actuar. ${ }^{13}$ En el contexto descrito, nuestro hospital y el sistema de salud conservaban la capacidad de contención y no había casos positivos aún en la institución.

\section{Análisis interno: nuestro Servicio}

Nuestro Servicio de Ortopedia y Traumatología se desempeña como tal en un hospital general de agudos del sistema público de salud de la CABA, en el área programática 01 que abarca segmentos de 5 de las 15 comunas porteñas, cuenta con una significativa aferencia de pacientes de una mayor área de influencia, así como de la colaboración con hospitales cercanos. Tiene una capacidad de 262 camas, dividida en pabellones ${ }^{25}$ La División de Ortopedia y Traumatología se encuentra organizada por equipos según subespecialidad que involucran Trauma, Columna Vertebral, Miembro Superior, Miembro Inferior, Cadera, Patología Traumatológica del Deporte y Artroscopia. Los cuadros prevalentes comprenden trauma, fracturas por osteoporosis y afecciones osteoarticulares degenerativas. 


\section{Directivas del Centro de Operaciones de Emergencia}

Nuestra estrategia de contingencia tenía la obligación de ser consonante con la normativa nacional y local dictada que llamaba a: ${ }^{6-12}$

- Organizarse en función de una posible emergencia.

- Disminuir las cirugías programadas.

- Aumentar las camas disponibles y, en especial, aquellas para aislamiento de pacientes con COVID-19.

- Considerar el refuncionamiento de sectores del hospital para contingencia de pacientes con COVID-19 y redistribución de pacientes.

- Prorrogar contratos de residentes y jefes de residentes.

- Otorgar licencias extraordinarias a viajeros a zonas de riesgo.

\section{Comité de Pandemia}

Se conformó el Comité de Pandemia de Ortopedia y Traumatología, debido a la necesidad de organizarse, basados en recomendaciones internacionales sobre cómo adaptar un servicio médico a la pandemia. ${ }^{26}$ Participan del comité: un traumatólogo, un médico clínico, un residente de traumatología, un enfermero y un instrumentador quirúrgico. Se designaron dos médicos a cargo exclusivamente del registro de datos y los protocolos de investigación. El objetivo principal del comité es elaborar e implementar una estrategia de contingencia que permita establecer lineamientos claros para la realización de prácticas médico-quirúrgicas en el contexto de la pandemia, controlar el correcto y eficaz uso de insumos, y el cumplimiento de las normas de bioseguridad. Además, funciona como nexo en la comunicación entre el comité de crisis del Hospital y el Servicio.

\section{Proceso: planificación, implementación y control}

El proceso fue dividido en tres instancias:

\section{A. Planificación: formulación de la estrategia}

\section{A.1. Etapa 1: búsqueda bibliográfica y clasificación de la evidencia}

Recolección de información actualizada sobre el tema, a cargo de los integrantes del comité, contemplando diversas fuentes de información: 1) bases de datos científicas: Medline, Embase, Cochrane, LILACS; 2) revistas nacionales e internacionales no indexadas, 3) guías de sociedades científicas nacionales e internacionales de Ortopedia y Traumatología, y Cirugía General, 4) sitios web de la Organización Mundial de la Salud y los Centros para el Control y la Prevención de Enfermedades de los Estados Unidos.

Para lograr una óptima comunicación entre los profesionales del Servicio, se propuso un programa de actualización diario mediante videoconferencias por Zoom (@2020 Zoom Video Communications), con reuniones coordinadas por el Comité de Pandemia y el jefe de División, con el objetivo de actualizar y exponer diariamente el análisis de la bibliografía en términos de nivel de evidencia, definición de problemas y estrategias de otros servicios. ${ }^{27}$

\section{A.2. Etapa 2: definir áreas de trabajo y equipos}

Análisis interno del Servicio y las características del recurso humano, para definir:

- Áreas de trabajo que hacen al funcionamiento del Servicio de Ortopedia y Traumatología.

- Profesionales a cargo de cada área con la finalidad de analizar la bibliografía y las normativas oficiales, enunciar problemas específicos del área y establecer estrategias de resolución.

Adicionalmente se solicitó a los jefes de las subespecialidades la confección de protocolos de diagnóstico y tratamiento de la patología más prevalente en nuestro medio, actualizando algoritmos al contexto de la pandemia de COVID-19 y definiendo las urgencias de cada subespecialidad.

\section{A.3. Etapa 3: escritura del documento}

Se encomendó al Comité de Pandemia la elaboración del documento, con posterior evaluación y corrección a cargo del jefe de División, el comité de crisis de la institución y el Director del Hospital.

\section{B. Implementación de la estrategia}

Se indicó la organización de las áreas de trabajo incluidas en la estrategia definiendo: responsables, objetivos y funciones. Coordinados por el Comité de Pandemia para reestructurar y señalizar espacios de trabajo, instruir y capacitar al personal de salud contemplando médicos, kinesiólogos, instrumentadores, enfermeros y administrativos del Servicio. 


\section{Control}

Se propuso desarrollar mecanismos de reporte al Comité de Pandemia sobre problemas o cambios en las normas vigentes durante la implementación.

\section{RESULTADOS}

\section{A. Planificación}

\section{A.1. Etapa 1: búsqueda bibliográfica y clasificación de la evidencia}

Realizamos la búsqueda bibliográfica en los motores de búsqueda PubMed y Google Académico con las siguientes palabras clave: "Coronavirus", "COVID-19", "Orthopedics", "Trauma", "Guidelines", "Pandemia", "Surgery", "Operating room", "Perioperative". Obtuvimos 20 artículos relacionados con nuestra especialidad, de los cuales excluimos a tres, porque eran descripciones aisladas o reportes de series breves de casos; en consecuencia, se seleccionaron 17 artículos. ${ }^{13,14,16,21-23,27-37}$

De los sitios web de sociedades científicas de Ortopedia y Traumatología, y Cirugía General, obtuvimos 13 documentos con recomendaciones y guías. ${ }^{15,17-20,38-45}$

En los sitios web de la Organización Mundial de la Salud y los Centros para el Control y la Prevención de Enfermedades de los Estados Unidos, seleccionamos 17 artículos con recomendaciones sobre aspectos relacionados con nuestra práctica. ${ }^{26,46-61}$

En las Tablas 1 y 2, se resumen los artículos y las guías de Ortopedia y Traumatología seleccionados, clasificados según el autor o la sociedad científica, la nacionalidad, el diseño del estudio, la temática y el nivel de evidencia.

Tabla 1. Bibliografía seleccionada (artículos)

\begin{tabular}{|c|c|c|c|c|c|}
\hline Autor & Fecha & Nacionalidad & Diseño & Temática & $\begin{array}{l}\text { Nivel de } \\
\text { Evidencia }\end{array}$ \\
\hline Dunham y cols. ${ }^{28}$ & 2020 & Estados Unidos & Revisión estándar & Conflictos bioéticos & IV \\
\hline Greenland y cols. ${ }^{29}$ & $10 / 03 / 2020$ & Estados Unidos & Revisión estándar & Perioperatorio y quirófano & IV \\
\hline Tang y cols. ${ }^{23}$ & 2020 & China & Consenso de expertos & Recomendaciones generales & IV \\
\hline Zheng y cols. ${ }^{30}$ & $03 / 2020$ & $\begin{array}{l}\text { China, Italia, } \\
\text { Austria }\end{array}$ & Consenso de expertos & Cirugía mininvasiva & IV \\
\hline Liang y cols. ${ }^{13}$ & 03/06/2020 & Singapur & Descriptivo & Recomendaciones generales & IV \\
\hline Prada y cols. ${ }^{22}$ & $12 / 04 / 2020$ & Canadá & Consenso de expertos & $\begin{array}{l}\text { Revisión exploratoria } \\
\text { (Scoping review) }\end{array}$ & IV \\
\hline Kogan y cols. ${ }^{31}$ & 2020 & Estados Unidos & Revisión estándar & Docencia & IV \\
\hline Massey y cols. ${ }^{21}$ & 2020 & Estados Unidos & Revisión estándar & Perioperatorio y quirófano & IV \\
\hline Awad y cols. ${ }^{32}$ & 2020 & Estados Unidos & Revisión estándar & Quirófano & IV \\
\hline Dexter y cols. ${ }^{33}$ & $24 / 03 / 2020$ & Estados Unidos & Revisión estándar & Perioperatorio y quirófano & IV \\
\hline Vaccaro y cols. ${ }^{16}$ & $03 / 2020$ & Estados Unidos & Revisión estándar & Recomendaciones generales & IV \\
\hline Wong y cols. ${ }^{34}$ & $04 / 03 / 2020$ & Canadá & Revisión estándar & Quirófano & IV \\
\hline Parisien y cols. ${ }^{27}$ & 2020 & Estados Unidos & Revisión estándar & Telemedicina. Docencia & IV \\
\hline $\begin{array}{l}\text { Vannabouathong } \\
\text { y cols. }{ }^{35}\end{array}$ & $01 / 04 / 2020$ & Canadá & Revisión estándar & Recomendaciones generales & IV \\
\hline Ti y cols. ${ }^{36}$ & 06/03/2020 & Canadá & Revisión estándar & Quirófano & IV \\
\hline $\begin{array}{l}\text { Rodriguez-Pinto } \\
\text { y cols. }{ }^{14}\end{array}$ & $03 / 2020$ & Portugal & Revisión estándar & Quirófano & IV \\
\hline Brindle y cols. ${ }^{37}$ & 2020 & Estados Unidos & Revisión estándar & $\begin{array}{l}\text { Manejo perioperatorio } \\
\text { Quirófano }\end{array}$ & IV \\
\hline
\end{tabular}


Tabla 2. Bibliografía seleccionada (guías)

\begin{tabular}{|c|c|c|c|c|}
\hline Sociedad & Fecha & Nacionalidad & Temática & Nivel de Evidencia \\
\hline $\mathrm{AAOS}^{38}$ & $31 / 03 / 2020$ & Estados Unidos & Manejo prequirúrgico & IV \\
\hline $\mathrm{ACS}^{19}$ & 2020 & Estados Unidos & Manejo de cirugías programadas & IV \\
\hline $\mathrm{SRC}^{20}$ & $20 / 03 / 2020$ & Reino Unido e Irlanda & Recomendaciones generales & IV \\
\hline SECOT $^{18}$ & $13 / 04 / 2020$ & España & Recomendaciones generales & IV \\
\hline $\mathrm{NHS}^{15}$ & $16 / 03 / 2020$ & Reino Unido & Manejo clínico- ortopédico & IV \\
\hline $\mathrm{AAOS}^{39}$ & $03 / 2020$ & Estados Unidos & Recomendaciones generales & IV \\
\hline $\mathrm{NHS}^{40}$ & $26 / 03 / 2020$ & Reino Unido & Recomendaciones generales & IV \\
\hline $\mathrm{CMS}^{41}$ & $07 / 04 / 2020$ & Estados Unidos & Manejos prequirúrgicos. Cirugías programadas & IV \\
\hline $\mathrm{AEC}^{42}$ & 2020 & España & Recomendaciones generales & IV \\
\hline $\mathrm{BOA}^{17}$ & $04 / 2020$ & Reino Unido & Manejo en urgencia & IV \\
\hline $\mathrm{ACC}^{43}$ & $26 / 03 / 2020$ & Colombia & Recomendaciones generales & IV \\
\hline $\mathrm{NHS}^{44}$ & $24 / 03 / 2020$ & Reino Unido & Manejo preoperatorio & IV \\
\hline $\mathrm{ACS}^{45}$ & $13 / 03 / 2020$ & Estados Unidos & Manejo preoperatorio & IV \\
\hline
\end{tabular}

AAOS = American Academy of Orthopedic Surgeons, ACS = American College of Surgeons, SRC = Surgical Royal Colleges of the United Kingdom and Ireland, SECOT = Sociedad Española de Ortopedia y Traumatología, NHS = National Health System, CMS = Centers for Medicare and Medicaid Services, $\mathrm{AEC}=$ Asociación Española de Cirugía, $\mathrm{BOA}=$ British Orthopaedic Association, $\mathrm{ACC}=$ Asociación Colombiana de Cirugía .

\section{A.2. Etapa 2: definir áreas de trabajo y equipos}

Se definieron 7 áreas de trabajo con un médico de planta a cargo: 1) Sala de internación, 2) Quirófano, 3) Consultorios externos, 4) Servicio de Urgencias, 5) Insumos, 6) Cuidado del personal de salud, 7) Docencia e investigación. Cada responsable de área definió la estrategia frente a los problemas específicos de cada área, según la bibliografía, las normas oficiales, nuestra estructura y recursos. La estrategia, según áreas de trabajo, se detalla en las Tablas 3-9.

Los jefes de subespecialidades redactaron los algoritmos diagnóstico y terapéutico de la patología más prevalente de nuestra institución, para adjuntar al protocolo.

\section{A.3. Etapa 3: escritura del documento}

Se redactó un documento, disponible para los profesionales del Servicio en forma digital e impresa, otorgando copias y acceso al Director del Hospital y al Comité de Crisis de la institución.

\section{B. Implementación}

Para su implementación se dictaron clases que incluían la lectura de la estrategia, la definición de normas de procedimiento y la capacitación del personal. Todas las charlas se realizaron online a través de la aplicación de videollamadas Zoom. Los profesionales de cada área conformaron equipos con un médico residente para elaborar elementos de señalización y reestructurar las áreas.

\section{Control}

Se definieron canales de comunicación con el Comité de Pandemia por videollamada telefónica, correo electrónico y reuniones diarias a través de la plataforma Zoom. Adicionalmente se pactaron revisiones semanales con la posibilidad de actualización. 
Tabla 3. Sala de internación ${ }^{15,16,18-20,22,31,38,44,45,48,49,55}$

- Programar una higiene regular, cada 3 h, de espacios comunes y lavado de superficies de alto contacto con soluciones antisépticas según la OMS.

- Señalizar y estimular el lavado de manos según la OMS.

- Definir médico referente de consulta (cargo rotativo) para resolver problemas, discutir indicaciones e interconsultas y programar cirugías, jerarquizando el tratamiento conservador.

- Minimizar los tiempos de internación pre- y posoperatorios.

- Definir criterios de internación priorizando urgencias.

- Utilizar Elementos de Protección Personal considerando: tipo de paciente (no expuesto, contacto estrecho, caso sospechoso/confirmado), portadores asintomáticos y sesgo de información en pacientes ancianos o no interrogables. Para curaciones y examen físico: camisolín de contacto, barbijo quirúrgico, guantes, máscara o antiparras. Ante producción de aerosoles o caso sospechoso/confirmado de COVID-19, adicionar respirador N95 y camisolín hemorrepelente.

- Asegurar el distanciamiento entre pacientes, en las habitaciones.

- Evaluar síntomas de COVID-19 y establecer protocolo de alarma y derivación.

- Limitar el ingreso de familiares.

- Suspensión de recorrida de sala presencial y evitar reuniones en sala, pasillos o enfermería.

- Devolución médica para familiares a través de videollamada.

- Reestructuración de la circulación del Servicio.

\section{Tabla 4. Quirófano ${ }^{13,15,16,18-23,29,30,32-34,37,38,41,42}$}

- Estadificar patología quirúrgica en: urgente (cirugía dentro de las 24 h de ingreso), urgente programada (cirugía a la brevedad) y programada (cirugía electiva). Suspender cirugías programadas.

- Minimizar el número de personas en el quirófano y la circulación.

- Minimizar los integrantes del equipo quirúrgico, priorizando tiempos y experiencia, mientras sea posible.

- Considerar técnicas quirúrgicas que han demostrado ser superiores significativamente al tratamiento conservador.

- No presenciar la intubación (aerosoles).

- Considerar procedimientos quirúrgicos de baja complejidad de insumos con resolución en el día.

- Conformar equipos quirúrgicos activos y de reserva con rotación bisemanal.

- Utilizar Elementos de Protección Personal considerando: tipo de paciente (no expuesto, contacto estrecho, caso sospechoso/confirmado), portadores asintomáticos, sesgo de información en pacientes ancianos o no interrogables, tipo de anestesia y filtros disponibles. Utilizar camisolín hemorrepelente, barbijo quirúrgico, guantes, antiparras y máscara. Adicionar respirador N95 si: producción de aerosoles, caso sospechoso/confirmado de COVID-19, paciente no interrogable, filtros de anestesia no aptos (humidificadores) en anestesia general. 


\section{Tabla 5. Consultorios externos ${ }^{15,18-22,40-42,52}$}

- Clasificar citas en: demorables (reprogramar su atención no generará secuelas en el paciente que lo sufre, patología tolerable y crónica) y urgentes (la demora puede producir secuelas permanentes). Reprogramar las consultas demorables.

- Minimizar el número de pacientes por agenda y en sala de espera.

- Minimizar los controles de seguimiento.

- Permitir el distanciamiento entre pacientes en la sala de espera.

- Señalizar la distancia de atención o las barreras físicas en el mesón de admisión.

- Interrogar sobre signos y síntomas de COVID-19 antes del ingreso en el área.

- Realizar un riguroso lavado de manos según la OMS.

- Distanciamiento físico durante el interrogatorio.

- Utilizar Elementos de Protección Personal considerando: tipo de paciente (no expuesto, contacto estrecho, caso sospechoso/ confirmado), portadores asintomáticos, sesgo de información en pacientes ancianos o no interrogables. Utilizar camisolín de contacto, barbijo quirúrgico, guantes, antiparras o máscara. Adicionar respirador N95 si: producción de aerosoles, caso sospechoso/confirmado de COVID-19, paciente no interrogable.

- Asegurar la ventilación del espacio de trabajo y la higiene de superficies de alto contacto con soluciones desinfectantes según la OMS.

- Minimizar las personas presentes en la consulta.

- Contemplar las herramientas de telemedicina para control y seguimiento.

- Minimizar la solicitud de estudios.

\section{Tabla 6. Servicio de Urgencia ${ }^{13-15,17-19,22}$}

- Organizar equipos de trabajo: activo y reserva, con rotación de acuerdo con las posibilidades.

- Evitar reuniones en espacios comunes: habitaciones, sala de médicos, enfermería.

- Utilizar llamadas/videollamadas para pases de guardia.

- Clasificar a los pacientes traumatizados según criterios de internación y posibilidad de control ambulatorio.

- Priorizar la resolución quirúrgica de urgencias.

- Optimizar los tratamientos ortopédicos, en especial, en fracturas pasibles de no ser operadas, pacientes pediátricos o comórbidos.

- Minimizar los controles radiográficos.

- Utilizar suturas absorbibles en heridas cortantes, cuando sea posible.

- Minimizar los controles ambulatorios después del alta.

- Realizar un riguroso lavado de manos según la OMS.

- Utilizar Elementos de Protección Personal considerando: tipo de paciente (no expuesto, contacto estrecho, caso sospechoso/confirmado), portadores asintomáticos, sesgo de información en pacientes ancianos o no interrogables. Utilizar camisolín de contacto, barbijo quirúrgico, guantes, antiparras y máscara. Adicionar respirador N95y camisolín hemorrepelente si: producción de aerosoles, caso sospechoso/confirmado de COVID-19, paciente no interrogable, manejo en sala de shock de politraumatizados graves. 


\section{Tabla 7. Insumos $22,48,58-61$}

- Contar y registrar los Elementos de Protección Personal disponibles.

- Estimar los Elementos de Protección Personal necesarios según la demanda y el monitoreo permanente.

- Reservar los Elementos de Protección Personal con acceso restringido y personal a cargo.

- Prevenir el uso innecesario de Elementos de Protección Personal.

- Establecer el tipo de Elementos de Protección Personal según el área.

- Establecer estrategias de respuesta frente a la falta de insumos.

- Registro de la entrega de respiradores N95 e instrucción al personal sobre: reutilización, conservación y descarte.

\section{Tabla 8. Control del personal de salud ${ }^{13,19,20,22,28,37,39,50,53,55}$}

- Realizar un seguimiento activo del riesgo de infección del personal a través de un cuestionario propuesto por la OMS, definir criterios de cuarentena o escudamiento.

- Dividir al personal de salud en equipos: activos y reserva con rotación semanal/bisemanal (de acuerdo con la disponibilidad).

- Registrar: no expuestos, sospechosos, infectados y rehabilitados.

- Considerar la reasignación de tareas priorizando mantener la capacidad quirúrgica de emergencia, proteger al equipo quirúrgico, capacitar en roles quirúrgicos no traumatológicos y en roles no quirúrgicos.

- Estratificar al personal por edad y factores de riesgo.

- Definir personal clave en roles quirúrgicos.

- Evitar conglomerados en zonas comunes: pasillos, salas de enfermería, aulas.

- Adecuar áreas de descanso según el distanciamiento.

- Estimular el lavado de manos y la higiene de superficies.

- Monitorear el estrés psicógeno del personal.

- Registro de lugares alternativos de trabajo (otros hospitales, clínicas o centros).

\section{Tabla 9. Docencia e investigación ${ }^{31,39,41}$}

- Suspender las actividades presenciales de carrera de especialista y cursadas de pregrado.

- Suspender la concurrencia de médicos (fellowships) o becarios no remunerados.

- Desarrollar un programa de formación de Residentes a través de videoconferencias.

- Asignar responsables en registro de datos y producción de protocolos de investigación en la pandemia.

- Desarrollar un programa de actualización de guías, normas y artículos relacionados con la pandemia.

- Desarrollar un programa de capacitación en tareas no relacionadas con la traumatología: consultorio de febriles, toma de muestras, conceptos de ventilación mecánica, triage. 


\section{DISCUSIÓN}

Desde el enfoque del trabajo, encontrar la descripción de experiencias y recomendaciones basadas en la evidencia, en la bibliografía fue muy complejo. El predominio de revisiones narrativas (estándares) y consensos de expertos deja notar la novedad del suceso, donde establecer, con rigurosidad metodológica, recomendaciones con un elevado nivel de evidencia resulta prácticamente imposible. Prada y cols. estiman esta problemática cuando, en una revisión bibliográfica (scoping review), solo un 5,3\% de los artículos fueron desarrollados con metodología basada en la evidencia. ${ }^{22}$

Algunos autores contaban con experiencia a partir de otras pandemias, como el caso de Liang, en Singapur, pero sin el nivel de diseminación y complejidad que el COVID-19 ha generado. ${ }^{13}$

Algunos autores de orígenes diversos coinciden en establecer prioridades similares, como el mantenimiento de la atención de las urgencias, la protección del personal y los pacientes, y la capacitación en roles alternativos. ${ }^{13-16,22}$

Destacamos que, independientemente de su peso estadístico, la información disponible fue un gran aporte ante la necesidad urgente de alcanzar una estrategia que guíe la toma de decisiones. Porque entendemos que el contexto de esta enfermedad, con su efecto a nivel global (pandemia), está en desarrollo, por eso nos encontramos con más interrogantes que certezas.

Cabe aclarar que, según nuestra opinión, el aporte de la bibliografía representa una etapa fundamental, pero no define "nuestra estrategia". Nuestra estrategia requirió: proponer un análisis interno y externo exhaustivo, definir nuestro medio y condición, definir nuestras fortalezas y debilidades como Servicio, definir nuestro rol predominante en el hospital y la comunidad, adaptar nuestra comunicación a la dinámica del proceso y estadificar las etapas de la pandemia por enfrentar.

Resulta muy difícil evaluar el desarrollo de un programa que se estructura e instala sobre la base de un acontecimiento masivo regional y global en tiempo "real" y donde el follow-up, por lo tanto, es nulo. Para los autores una forma de autopercibirse cuando las cosas están funcionando bien "en el terreno", es observar el grado de cohesión del grupo, comparativamente con su funcionamiento habitual o de rutina, y su funcionamiento más o menos caótico ante la implementación de las medidas que se toman. Destacamos dos elementos fundamentales en esta situación especial: la división horizontal de funciones y la comunicación efectiva cotidiana con todos los miembros del equipo de salud para actualizar los conocimientos y la problemática asegurando la eficaz toma de decisiones.

\section{CONCLUSIONES}

Nuestro trabajo aporta una propuesta para el proceso de desarrollo de una estrategia de contingencia frente a la pandemia de COVID-19. Organizarse, capacitarse y protegerse como equipo son sus pilares fundamentales. Todos los recursos, desde la información hasta el ingenio y la fortaleza que nos caracteriza a los traumatólogos, son necesarios, especialmente en el contexto de un hospital público.

Conflicto de intereses: Los autores no declaran conflictos de intereses.

ORCID de M. Pérez: https://orcid.org/0000-0002-2163-0680 ORCID de S. Scalambro: https://orcid.org/0000-0002-7478-0041 ORCID de G. Ricciardi: https://orcid.org/0000-0002-6952-7260
ORCID de G. Carrioli: https://orcid.org/0000-0003-4160-9712 ORCID de F. Locaso: https://orcid.org/0000-0003-2248-5582 ORCID de D. Ricciardi: https://orcid.org/0000-0002-1396-9115

\section{BIBLIOGRAFÍA}

1. World Health Organization Press Conference. WHO Director-General's opening remarks at the media briefing on COVID-19 - 11 March 2020. Disponible en: https://www.who.int/es/dg/speeches/detail/who-director-general-sopening-remarks-at-the-media-briefing-on-covid-19---11-march-2020. Consulta: 11 de marzo, 2020.

2. Tan WJ, Zhao X, Ma XJ, Wang W, Niu P, X W, et al. A novel coronavirus genome identified in a cluster of pneumonia cases-Wuhan, China 2019-2020. China CDC Weekly 2020;2(4):61-2.

https://doi.org/10.46234/cedcw2020.017 
3. Zhu N, Zhang D, Wang W, Li X, Yang B, Song J, et al. A novel coronavirus from patients with pneumonia in China, 2019. N Engl J Med 2020;382:727-33. https://doi.org/10.1056/NEJMoa2001017

4. Ministerio de Salud de la República Argentina. Nuevo Coronavirus (COVID19). Reporte Diario. Disponible en: https://www.argentina.gob.ar/sites/default/files/5-03-2020-nuevo-coronavirus-covid-19-reporte-diario_1.pdf. Consulta: 5 de marzo, 2020.

5. Ministerio de Salud de la República Argentina. Nuevo Coronavirus (COVID19). Reporte Diario. Disponible en: https://www.argentina.gob.ar/sites/default/files/12-03-20-nuevo-coronavirus-covid-19_reporte-diario_0.pdf. Consulta: 12 de marzo, 2020.

6. Decreto 260/2020. Emergencia Sanitaria - Coronavirus (COVID-19). Boletín Oficial de la República Argentina. DECNU-2020-260-APN-PTE. 12/03/2020. Disponible en: https://www.boletinoficial.gob.ar/suplementos/2020031201NS.pdf. Consulta: 12 de marzo, 2020.

7. Ministerio de Salud de la República Argentina. Plan Operativo de preparación y respuesta al COVID-19. Disponible en: https://www.argentina.gob.ar/salud/coronavirus-COVID-19/plan-operativo. Consulta: 12 de marzo, 2020.

8. Gobierno de la Ciudad Autónoma de Buenos Aires. Directivas Centro de Operaciones de Emergencia COVID19. ME-2020-09911443 GCABA-DGHOS. 18/03/2020. Disponible en: https://eaaf.org/wp-content/uploads/covid19-PDFs/Argentina/IF-2020-10223708-GCABA-DGHOSP.pdf. Consulta: 19 de marzo, 2020.

9. Gobierno de la Ciudad Autónoma de Buenos Aires. Proyecto de resolución. EE-2020-18074310-APN-DNCH\#MS Prórroga Promoción. Poder Ejecutivo Nacional. República Argentina. Ciudad de Buenos Aires. 19/03/20.

10. Gobierno de la Ciudad Autónoma de Buenos Aires. DECAD-2020-371-APN-JGM - Licencia excepcional. Coronavirus (COVID-19). BO. Suplemento 12/03/2020. Disponible en: https://www.boletinoficial.gob.ar/suplementos/2020031201NS.pdf. Consulta: 16 de marzo, 2020.

11. Ministerio de Salud de la República Argentina. Contratación por emergencia COVID19 N²/20. EX-202021915903--APN-SSGA\#MS. Poder Ejecutivo Nacional. 03/04/2020. Disponible en: https://www.argentina.gob.ar/salud/contratacion-por-emergencia-covid-19. Consulta: 4 de abril, 2020.

12. Gobierno de la Ciudad Autónoma de Buenos Aires. ME-2020-11683110-GCABA-DGHOS. Reasignación de funciones y redistribución del personal COVID-19. 16/04/2020. Disponible en: http://www.hdurandinfo.com/. Consulta: 17 de abril, 2020.

13. Liang ZC, Wang W, Murphy D, Hui JHP. Novel coronavirus and orthopaedic surgery: early experiences from Singapore. J Bone Joint Surg Am 2020;e000236. https://doi.org/10.2106/JBJS.20.00236

14. Rodriguez-Pinto R, Sousa R, Oliveira A. Preparing to perform trauma and orthopaedic surgery on patients with COVID-19. J Bone Joint Surg Am 2020;e20.00454. https://doi.org/10.2106/JBJS.20.00454

15. NHS England and NHS Improvement. Clinical guide for the management of trauma and orthopaedic patients during the coronavirus pandemic. Disponible en: https://www.england.nhs.uk/coronavirus/wp-content/uploads/ sites/52/2020/03/C0274-Specialty-guide-Orthopaedic-trauma-v2-14-April.pdf. Consulta: 14 de abril, 2020.

16. Vaccaro AR, Getz CL, Cohen BE, Cole BJ, Donnally CJ 3rd. Practice management during the COVID-19 pandemic. J Am Acad Orthop Surg 2020;28(11):464-70. https://doi.org/10.5435/JAAOS-D-20-00379

17. British Orthopaedic Association. Management of patients with urgent orthopaedic conditions and trauma during the coronavirus pandemic. Disponible en: https://www.boa.ac.uk/resources/covid-19-boasts-combined.htm. Consulta: 13 de abril, 2020.

18. SECOT. Recomendaciones Generales de la Sociedad Española de Cirugía Ortopédica y Traumatología frente al COVID-19. Disponible en: https://www.secot.es/media/docs/covid19/RecomendacionesSECOTGeneralesDeCOTFrenteAlCovid19.pdf. Consulta: 13 de abril, 2020.

19. COVID-19 Guidelines for Triage of Orthopaedic Patients. American College of Surgeons. Disponible en: https://www.facs.org/covid-19/clinical-guidance/elective-case/orthopaedics. Consulta: 4 de abril, 2020.

20. Guidance for surgeons working during the COVID-19 pandemic from the Surgical Royal Colleges of the United Kingdom and Ireland. Disponible en: https://www.rcseng.ac.uk/coronavirus/joint-guidance-for-surgeons-v1/. Consulta: 20 de marzo, 2020.

21. Massey PA, McClary K, Zhang AS, Savoie FH, Barton RS. Orthopaedic surgical selection and inpatient paradigms during the coronavirus COVID-19 pandemic. J Am Acad Orthop Surg 2020;28(11):436-50. https://doi.org/10.5435/JAAOS-D-20-00360

22. Prada C, Chang Y, Poolman R, Johal H, Bhandari M. Best Practices for Surgeons -COVID-19 Evidence-Based Scoping Review. A unifying report of global recommendations. Disponible en: https://aaot.org.ar/wp-content/ uploads/2020/04/OE-Best-Practices-for-Surgeons-COVID-19-Evidence-Based-Scoping-Review.pdf_compressed1.pdf. Consulta: 4 de abril 2020. 
23. Tang PF, Hou ZY, Wu XB, Zhang CQ, Wang JW, Xing X, et al. Expert consensus on management principles of orthopedic emergency in the epidemic of Corona Virus Disease 2019. Chin Med J 2020;133(9):1096-8. https://doi.org/10.1097/CM9.0000000000000810

24. Decreto 297/2020. Aislamiento Social Preventivo y Obligatorio. Boletín Oficial de la República Argentina. DECNU-2020-297-APN-PTE - Disposiciones. 19/03/2020. Disponible en: https://www.boletinoficial.gob.ar/detalleAviso/primera/227042/20200320. Consulta: 20 de marzo, 2020.

25. Gobierno de la Ciudad Autónoma de Buenos Aires. Áreas programáticas de la Salud. 2016. Disponible en: https://www.estadisticaciudad.gob.ar/eyc/wp-content/uploads/2016/08/SA071602.pdf. Consulta: 20 de marzo, 2020.

26. Center for Disease Control and Prevention (CDC). Steps Healthcare Facilities Can Take Now to Prepare for COVID-19. Disponible en: https://www.cdc.gov/coronavirus/2019-ncov/hcp/steps-to-prepare.html. Consulta: 20 de marzo, 2020.

27. Parisien RL, Shin M, Constant M, Saltzman BM, Li X, Levine WN, et al. Telehealth utilization in response to the novel coronavirus (COVID-19) pandemic in orthopaedic surgery. J Am Acad Orthop Surg 2020. https://doi.org/10.5435/JAAOS-D-20-00339

28. Dunham AM, Rieder TN, Humbyrd CJ. A bioethical perspective for navigating moral dilemmas amidst the COVID-19 pandemic. J Am Acad Orthop Surg 2020;28(11):471-6. https://doi.org/10.5435/JAAOS-D-20-00371

29. Greenland JR, Michelow MD, Wang L, London MJ. COVID-19 Infection implications for perioperative and critical care physicians. Anesthesiology 2020;132(6):1346-61. https://doi.org/10.1097/ALN.0000000000003303

30. Zheng MH, Boni L, Fingerhut A. Minimally invasive surgery and the novel coronavirus outbreak: lessons learned in China and Italy. Ann Surg 2020. https://doi.org/10.1097/SLA.0000000000003924

31. Kogan M, Klein SE, Hannon CP, Nolte MT. Orthopaedic education during the COVID-19 pandemic. J Am Acad Orthop Surg 2020;28(11):e456-e464. https://doi.org/10.5435/JAAOS-D-20-00292

32. Awad ME, Rumley JC, Vazquez JA, Devine JG. Peri-operative considerations in urgent surgical care of suspected and confirmed COVID-19 orthopedic patients: operating rooms protocols and recommendations in the current COVID-19 pandemic. J Am Acad Orthop Surg 2020;28(11):451-63. https://doi.org/10.5435/JAAOS-D-20-00227

33. Dexter F, Parra MC, Brown JR, Loftus RW. Perioperative COVID-19 defense: an evidence-based approach for optimization of infection control and operating room management. Anesth Analg 2020. https://doi.org/10.1213/ANE.0000000000004829

34. Wong J, Goh QY, Tan Z, Lie SA, Tay YC, Ng SY, et al. Preparing for a COVID-19 pandemic: a review of operating room outbreak response measures in a large tertiary hospital in Singapore. Can J Anesth/J Can Anesth 2020;67: 732-45. https://doi.org/10.1007/s12630-020-01620-9

35. Vannabouathong C, Devji T, Ekhtiari S, Chang Y, Phillips SA, Zhu M, et al. Novel coronavirus COVID-19: current evidence and evolving strategies. J Bone Joint Surg Am 2020. https://doi.org/10.2106/JBJS.20.00396

36. Ti LK, Ang LS, Foong TW, Ng BSW. What we do when a COVID-19 patient needs an operation: operating room preparation and guidance. Can J Anaesth 2020;67(6):756-8. https://doi.org/10.1007/s12630-020-01617-4

37. Brindle M, Gawande A. Managing COVID-19 in surgical systems. Ann Surg 2020. https://doi.org/10.1097/SLA.0000000000003923

38. Guy DK, Bosco JA, Savoie FH. AAOS Guidelines on Elective Surgery during the COVID-19 Pandemic: March 31. COVID-19: Member Resource Center. Disponible en: https://www.aaos.org/globalassets/about/covid-19/aaos-guidelines-on-electivesurge.pdf. Consulta: 3 de abril, 2020.

39. American Academy of Orthopaedic Surgeons. Clinical considerations during COVID19. Disponible en: https://www.aaos.org/globalassets/about/covid-19/aaos-clinical-considerations-during-covid-19.pdf. Consulta: 12 de abril, 2020.

40. NHS England and NHS Improvement. Redeploying your secondary care medical workforce safely. Disponible en: https://www.england.nhs.uk/coronavirus/wp-content/uploads/sites/52/2020/03/Redeploying-your-secondary-caremedical-workforce-safely_26-March.pdf. Consulta: 26 de marzo, 2020.

41. CMS's recommendations for adult elective surgery and procedures. Disponible en: https://www.aaos.org/about/ covid-19-information-for-our-members/aaos-guidelines-for-elective-surgery/. Consulta: 26 de marzo, 2020.

42. Documentos de posicionamiento y recomendaciones de la AEC en relación con la cirugía y COVID-19. Disponible en: https://www.aecirujanos.es/Documentos-de-posicionamiento-y-recomendaciones-de-la-AEC-en-relacion-con-lacirugia-y-COVID19_es_1_152.html. Consulta: 26 de marzo, 2020.

43. Guidelines on Delaying Cancer Surgery during COVID-19. Disponible en: https://www.medscape.com/viewarticle/927568_print. Consulta: 26 de marzo, 2020.

44. NHS England and NHS Improvement. Clinical guide for the perioperative care of people with fragility fractures during the Coronavirus pandemic. Disponible en: https://www.boa.ac.uk/uploads/assets/3e2ef4d7-6788-4fcd8a17196fca00f63e/NHSE-Fragility-Fractures-and-Coronavirus-VBOA10-24032020docx.pdf. Consulta: 23 de marzo, 2020. 
45. American College of Surgeons. COVID-19: Recommendations for Management of Elective Surgical Procedures. Disponible en: https://www.facs.org/-/media/files/covid19/recommendations_for_management_of_elective_surgical_procedures.ashx. Consulta: 23 de marzo, 2020.

46. World Health Organization. Critical preparedness, readiness and response actions for COVID-19. Interim guidance, 22 March 2020. Disponible en: https://www.who.int/publications-detail/critical-preparedness-readiness-and-response-actions-for-covid-19. Consulta: 26 de marzo, 2020.

47. World Health Organization. Rational use of personal protective equipment for coronavirus disease (COVID-19): interim guidance, 27 February 2020. Disponible en: https://apps.who.int/iris/handle/10665/331215. Consulta: 12 de marzo, 2020.

48. World Health Organization. Rational use of personal protective equipment for coronavirus disease ("COVID-19)" and considerations during severe shortages: interim guidance, 6 April 2020. Disponible en: https://www.who. int/publications-detail/rational-use-of-personal-protective-equipment-for-coronavirus-disease-(covid-19)-andconsiderations-during-severe-shortages. Consulta: 12 de abril, 2020.

49. World Health Organization. Infection prevention and control during health care when novel coronavirus (nCoV) infection is suspected: interim guidance, 19 March 2020. Disponible en: https://www.who.int/publications-detail/ infection-prevention-and-control-during-health-care-when-novel-coronavirus-(ncov)-infection-is-suspected-20200125. Consulta: 20 de marzo, 2020.

50. World Health Organization. Health workers exposure risk assessment and management in the context of COVID-19 virus: interim guidance, 4 March 2020. Disponible en: https://apps.who.int/iris/handle/10665/331340. Consulta: 20 de marzo, 2020.

51. Centers for Disease Control and Prevention (CDC). Use Personal Protective Equipment (PPE). Disponible en: https://www.cdc.gov/coronavirus/2019-ncov/hcp/using-ppe.html. Consulta: 3 de abril, 2020.

52. Centers for Disease Control and Prevention (CDC). Outpatient and Ambulatory Care Settings: Responding to Community Transmission of COVID-19 in the United States. Disponible en: https://www.cdc.gov/coronavirus/2019-ncov/hcp/ambulatory-care-settings.html. Consulta: 7 de abril, 2020.

53. Centers for Disease Control and Prevention (CDC). Healthcare Infection Prevention and Control FAQs for COVID-19. Operational Considerations for the Identification of Healthcare Workers and Inpatients with Suspected COVID-19 in non-US Healthcare Settings. Disponible en:

https://www.cdc.gov/coronavirus/2019-ncov/hcp/non-us-settings/guidance-identify-hcw-patients.html. Consulta: 6 de abril, 2020.

54. Centers for Disease Control and Prevention (CDC). Interim Infection Prevention and Control Recommendations for Patients with Suspected or Confirmed Coronavirus Disease 2019 (COVID-19) in Healthcare Settings. Disponible en: https://www.cdc.gov/coronavirus/2019-ncov/hcp/infection-control-recommendations.html. Consulta: 12 de abril, 2020.

55. Centers for Disease Control and Prevention (CDC). Interim U.S. Guidance for Risk Assessment and Public Health Management of Healthcare Personnel with Potential Exposure in a Healthcare Setting to Patients with Coronavirus Disease 2019 (COVID-19). Disponible en: https://www.cdc.gov/coronavirus/2019-ncov/hcp/guidance-risk-assesment-hcp.html. Consulta: 12 de abril, 2020.

56. Centers for Disease Control and Prevention (CDC). Strategies for Optimizing the Supply of N95 Respirators during the COVID-19 Response. Disponible en: https://www.cdc.gov/coronavirus/2019-ncov/hcp/respirators-strategy/index.html. Consulta: 12 de abril, 2020.

57. Centers for Disease Control and Prevention (CDC). Steps Healthcare Facilities Can Take Now to Prepare for COVID-19. Disponible en: https://www.cdc.gov/coronavirus/2019-ncov/hcp/steps-to-prepare.html. Consulta: 20 de marzo, 2020.

58. Centers for Disease Control and Prevention (CDC). Strategies for Optimizing the Supply of Eye Protection. Disponible en: https://www.cdc.gov/coronavirus/2019-ncov/hcp/ppe-strategy/eye-protection.html. Consulta: 17 de marzo, 2020.

59. Centers for Disease Control and Prevention (CDC). Strategies for Optimizing the Supply of Facemasks. Disponible en: https://www.cdc.gov/coronavirus/2019-ncov/hcp/ppe-strategy/face-masks.html. Consulta: 17 de marzo, 2020.

60. Centers for Disease Control and Prevention (CDC). Strategies for Optimizing the Supply of Isolation Gowns. Disponible en: https://www.cdc.gov/coronavirus/2019-ncov/hcp/ppe-strategy/isolation-gowns.html. Consulta: 17 de marzo, 2020.

61. Centers for Disease Control and Prevention (CDC). Strategies to Mitigate Healthcare Personnel Stang Shortages. Disponible en: https://www.cdc.gov/coronavirus/2019-ncov/hcp/mitigating-staff-shortages.html. Consulta: 13 de abril, 2020. 